\title{
STRESS ANALYSIS OF TURBINE COMPONENTS UNDER SPIN RIG THERMOMECHANICAL CONDITION
}

\section{Witek}

\author{
Rzeszow University of Technology, Department of Mechanical Engineering and Aeronautics; \\ 35-959 Rzeszow, Powstancow Warszawy Ave. 8; Poland; E-mail: lwitek@prz.rzeszow.pl \\ Received 1012 2004, accepted 13122004
}

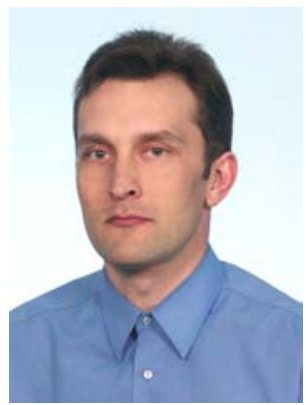

\begin{abstract}
Lucjan WITEK, Ph.D. Eng.
Was born in 1972. Graduated in 1997 with M.Sc. degree in mechanical engineering (specialization: aerospace), Ph.D. degree in 2002 (discipline: mechanics), both from the Rzeszow University of Technology, Poland. Currently assistant professor at Department of Mechanical Engineering and Aeronautics of Rzeszow University of Technology. Research focuses on fatigue and fracture studies, non-linear stress analysis of mechanical and aircraft structures, and analysis of stability of thin-walled structures. Since 1997 has worked as a university lecturer in the field of strength of material, strength of aircraft structures, and finite element method. In 2003 given NATO Fellowship Organization award for project concerned with FE analysis of stress, fatigue life, and reliability of hot and cold sections of gas turbine engine components. In 09.2003-06.2004 participant of NATO Postdoctoral Fellowship at Institute for Aerospace Research; National Research Council-Canada, Ottawa
\end{abstract}

Abstract. This paper presents the stress analysis of a second stage turbine disc, subjected to spin rig test. To solve the problem, the finite element analysis of complex geometrical model of the disc/blade segment with much physical nonlinearity such as contact and plasticity was carried out. The adequate mechanical and thermal conditions for the spin rig test were next defined to determine the stress state of the disc/blade segment. High stress zones were found at the regions of the turbine where the first fatigue cracks are expected to appear. The results of this study are useful during the laboratory spin rig test of turbine. The zones of maximum stress indicated in this analysis will be in detail inspected under laboratory crack initiation and crack growth tests of turbine. For better understanding the phenomena occurring in spin rig test of turbine, the test details were also described.

Keywords: spin rig test, stress analysis, turbine disc, aircraft engine, FEM.

\section{Introduction}

Before gas turbine engine components are released into service, they are usually tested in simulated engine conditions, mainly in a spin rig environment. This testing is performed during the component development stage and during certification and improvement stages of the gas turbine engine development process. The spin rig test could also be performed under a life extension study to estimate the real fatigue life of hot section engine components.

The life extension studies with use the "damage tolerance" philosophy are nowadays performed for aircraft, due to the fact that the cost of a credible life extension study is much lower then the cost of a new aircraft. The damage-tolerance approach to enginemaintenance policies establishes that components could be inspected periodically and undamaged parts put back into service for a specific number of flight hours. Saving engines from retirement leads to substantial savings for aircraft operators.

Typical tests performed in a spin rig involve crack initiation tests, crack growth tests, and burst tests. All these tests are performed in isothermal conditions, which means that the component tested is held at a constant temperature during the entire period of a test.

The real gas turbine discs works mostly at high temperature gradients and is subjected to high rotational velocity. To compensate for a lack of thermal gradient in the component tested, various adjustments to the test parameter are made, such as adjusting the component rotational speed or changing the test temperature. These adjustments are generally supported by calculations. In addition, they are based on strong assumptions of the failure modes of the tested components. This approach introduces severe uncertainty to the test results, contributes to difficulty in the interpretation of the results, and also increases testing costs. A several tests have to be performed to target crack initiation or crack growth at different locations on the component.

The service life of critical aerospace components is governed by several modes of degradation and failure such as: fatigue, fracture, yielding, creep, corrosion, erosion, and wear etc. Gas turbine discs are usually the most critical engine components and must endure substantial mechanical and thermal loading.

If a problem arises in the turbine section, it will significantly affect the whole engine function and, of course, the safety of the aircraft. Blade loss can be contained within the engine casing, while the catastrophic failure of a turbine wheel, can cause the larger fragments of the disc to puncture the engine casing (Figure 1). In spin rig tests, just this kind of failure can be safely simulated and investigated. 


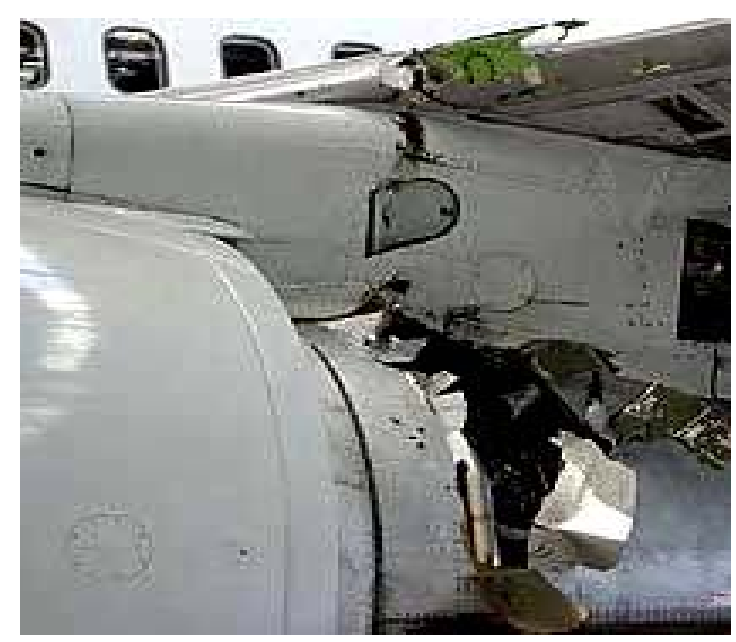

a)

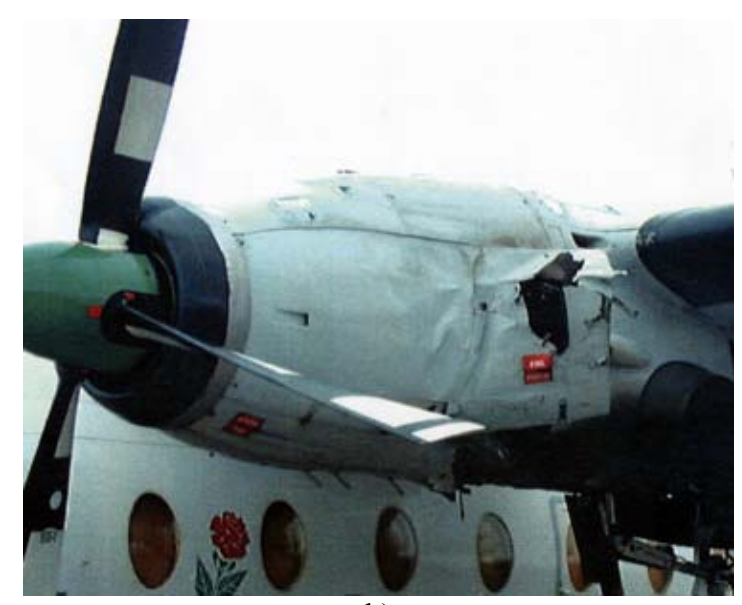

b)

Fig 1. The engine casings punctured by disc fragments after catastrophic failure of turbine
The results of the stress analysis of turbine components that work in spin rig conditions have not been widely described in literature. Of interest is a work by Zhuang, in which the author describes the investigation concerned with the analysis of crack growth of a turbine disc under spin rig conditions [13]. The problem of numerical evaluation of the stress state of a disc and Chan at al. describes blade subjected to real engine thermo mechanical conditions, Masataka, Meguid, Papanikos, and Zboinski [4, 8, 11, 12]. Also of interest is a work written by McEvity, in which the author analyzes the failures of engines used to power MD-88, DC-10 and B737 planes [6]. Hou described the experimental and numerical investigation of phenomenon occurring in the fatigue fracture of turbine blades [5]. Bhaumik and Park also described the problem of fatigue fracture of turbine components at al. [3, 10].

In this study, attention is devoted to the analysis of the stress state of second stage turbine components subjected to spin rig conditions. The spin rig facility and difficulties occurred during the spin rig tests were also described in this paper.

\section{Spin rig test facility}

Spin testing is an important step in the prevention of centrifugal burst disaster. Rotating components used in a high-speed turbine operate under large centrifugal stresses and can fail with explosive force. All designers and manufacturers of turbo machinery components therefore need to test rotors for centrifugal strength. Designers need to calibrate and verify the results of stress analysis and establish the fatigue life of high-performance turbines. Manufacturers also need to prove the strength of highspeed disks before shipment and installation.

The spin rig test facility is showed in Figure 2.

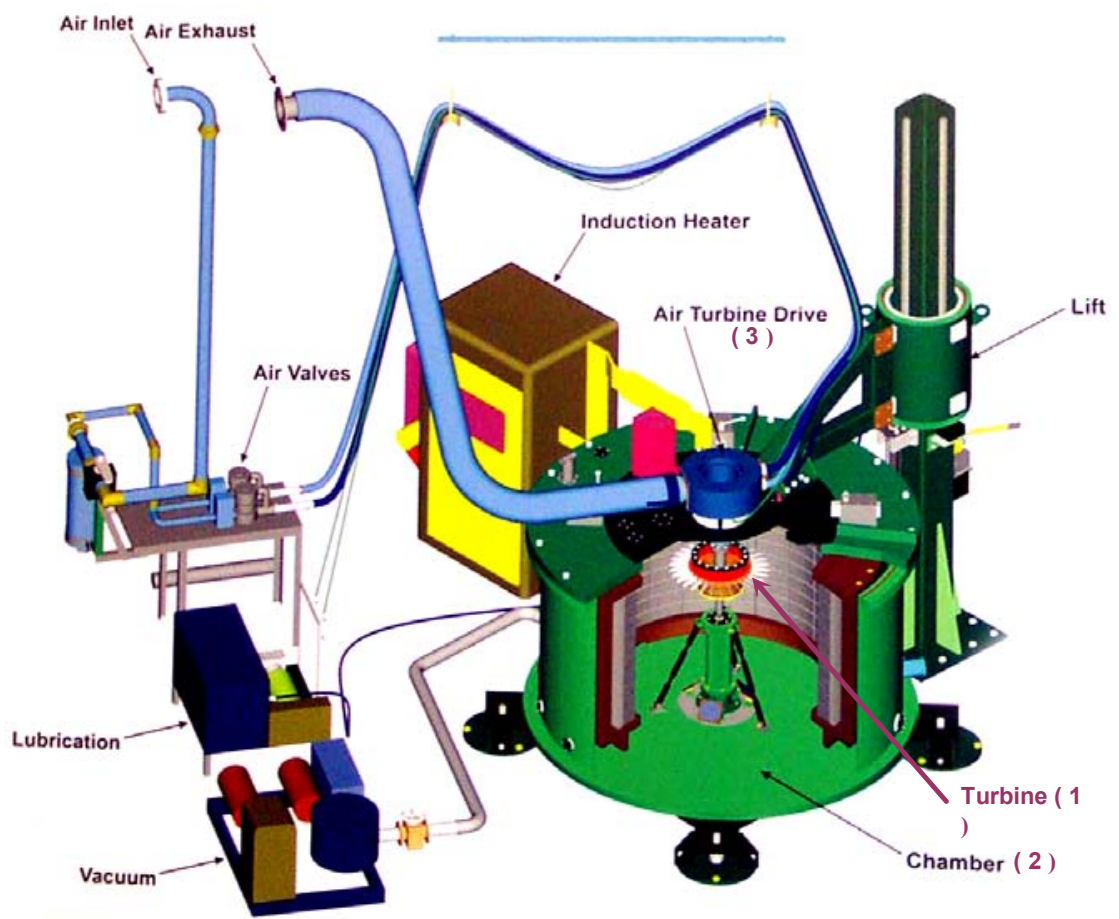

Fig 2. Spin rig facility 
The most common way to conduct a spin test is to suspend a turbine (1) in a heavily armored vacuum chamber (2) from a flexible spindle and to accelerate the rotor to high speed by driving the spindle with a compressed air turbine (3). The flexible shaft allows the rotor to find its own axis of balance. The soft support eliminates the need for extreme precision in mounting or balancing and limits the damage to the drive system in the event of a rotor burst. A high strength forged steel cylinder with a minimum thickness equal to $1 / 3$ of the radius of the largest diameter test rotor is appropriate for most common applications. To reduce the magnitude of impact shock, the steel armor is commonly lined with a soft material like lead, which can absorb a lot of energy under disc burst.

Spin tests are usually conducted in vacuum to eliminate the high air friction losses that would make it difficult to drive most rotors at high speed and to reduce the friction heating created if the test were conducted in air. The vacuum is an important safety feature since it reduces the risk of explosion of metal dust or oil fog during rotor failure. It is also important in preventing unsteady aerodynamic forces from destabilizing the rotor. The necessary vacuum is at about $1 / 4000$ of absolute atmospheric pressure.

The low power of the traditional air turbine test drives has been a handicap in the measurement of cyclic fatigue life. With the old drives, cycle times measured in minutes were common, and a 50,000 cycle life test required five months of continuous test operation. Modern high-efficiency air turbines reduce the cycle time to 10-30 seconds for typical jet engine rotors. It allows thousands of flight hours of engine life to be compressed into several weeks.

Spin testing is potentially dangerous. The kinetic energy of typical turbo machinery components is very high at burst speed, and the fragments of a disk can do serious damage to structures and people if containment is inadequate. To endure the impact of turbine fragments, the inside surface of the rig chamber is covered by thick bricks made of lead. The rig chamber with turbine prepared for investigation is shown in Figure 3.

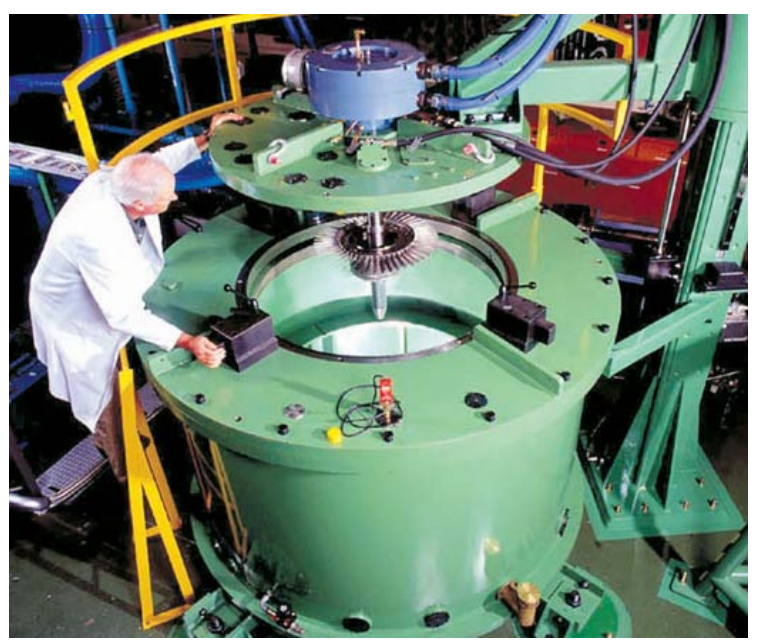

Fig 3. View of rig chamber
The analysis presented here was made as a preliminary investigation, before performing the laboratory spin rig test of turbine. For this reason, the results of laboratory turbine spin test are not included in this work.

\section{Numerical FE simulation of spin rig test of turbine}

In the simulation presented here, the detailed geometric model of 1/89 turbine segment (one segment from 89 repetitive segments of turbine) was first defined.

The constructional surfaces used to create this model are shown in Figure 4.

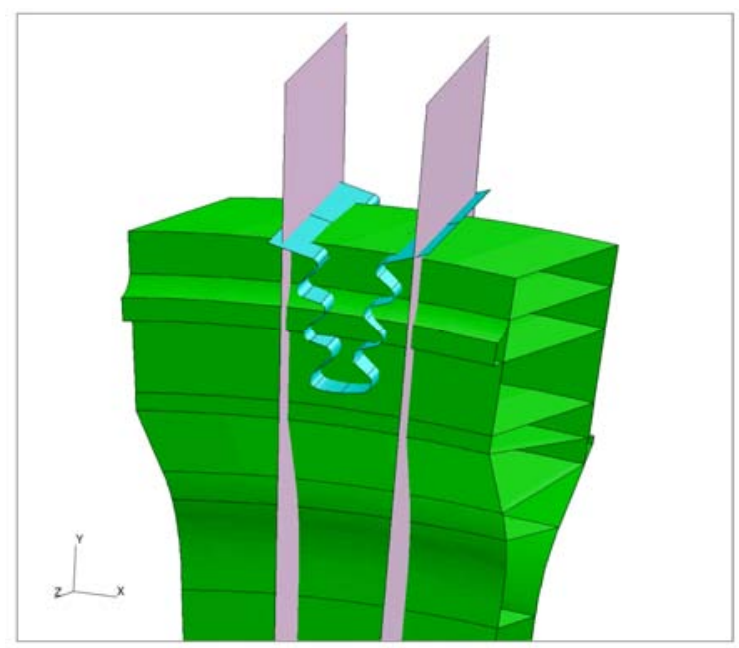

Fig 4. Border surfaces of disc used to create the model of geometry

The special boundary condition (known as "cyclic symmetry") was defined to modeling the interaction between repetitive border surfaces of disc (Figure 4) [1]. These surfaces are rotated at $4,045^{\circ}$ around the disc axis symmetry.

The finite element program MSC-Patran 2004 was used to create both the geometric and discrete models of the disc segment and blade [9]. The finite element model of the turbine presented in Fig 5a consists of 26738 solid elements (Hex-8 and Tet-4) [9]. To model the mechanical interface of adjacent surfaces of the disc and blade (Figure 5b), the "master-slave" type of contact with friction coefficient of 0.1 was defined [1].

In this analysis a constant thermal load of $550^{\circ} \mathrm{C}$ was applied to all nodes of the FE model. To compensate for the lack of thermal gradient in the component tested, the rotational speed of turbine was elevated from 13800 $\mathrm{rpm}$ (operational speed of engine turbine) to $14100 \mathrm{rpm}$.

The second stage turbine disc is manufactured out of Wasp alloy material. This alloy is a precipitationhardened nickel-base super alloy with good strength, ductility, and fracture toughness over a temperature range of -200 to $700{ }^{\circ} \mathrm{C}$. These properties, along with good weld ability and formability account, for its wide use in aerospace applications. The yield point of Waspalloy (for $550^{\mathrm{O}}$ ) is $1250 \mathrm{MPa}$, while the UTS (ultimate tensile strength) is $1400 \mathrm{MPa}$. 


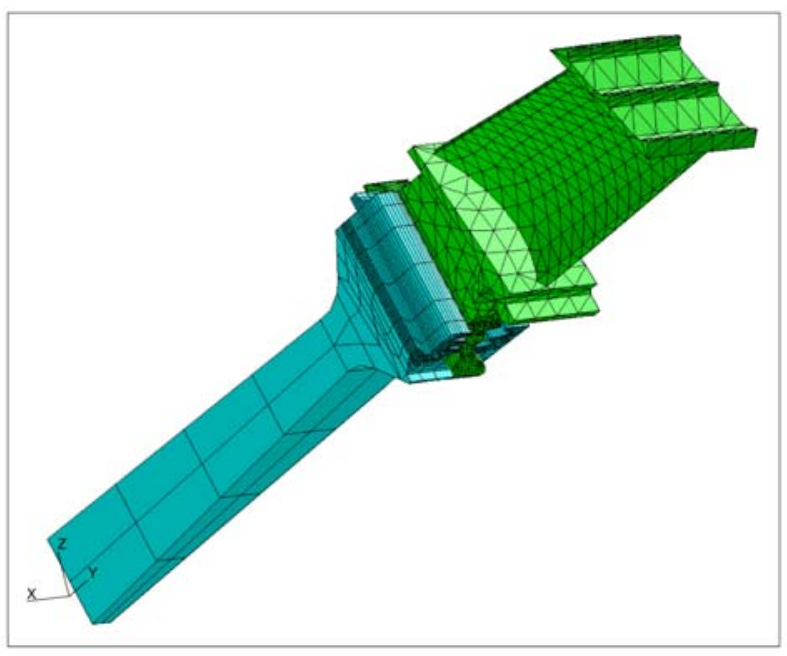

a)

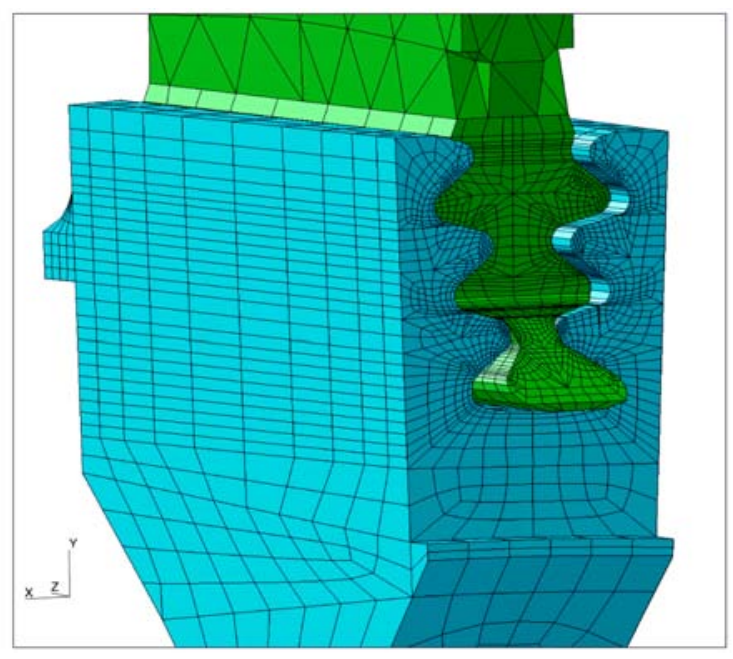

b)

Fig 5. Finite element model of the turbine segment (a) and mesh in the vicinity of fir-tree slots (disc-blade connection) (b)

The blades for the second stage turbine are manufactured out of MAR-247 material. This alloy is a precipitation-hardened nickel base superalloy, with better creep resistance in a high temperature than Waspalloy. This numerical analysis was performed for isotropic hardened elastic-plastic disc and blade materials.

\section{Results of finite element simulations}

The ABAQUS v. 6.4 solvers was used for stress analysis of the second stage turbine disc. In the calculation reported here the nonlinear Newton-Raphson method was applied. For all results presented below, the Megapascals (MPa) and millimeters ( $\mathrm{mm}$ ) were used to describe the fields of stresses and displacements.

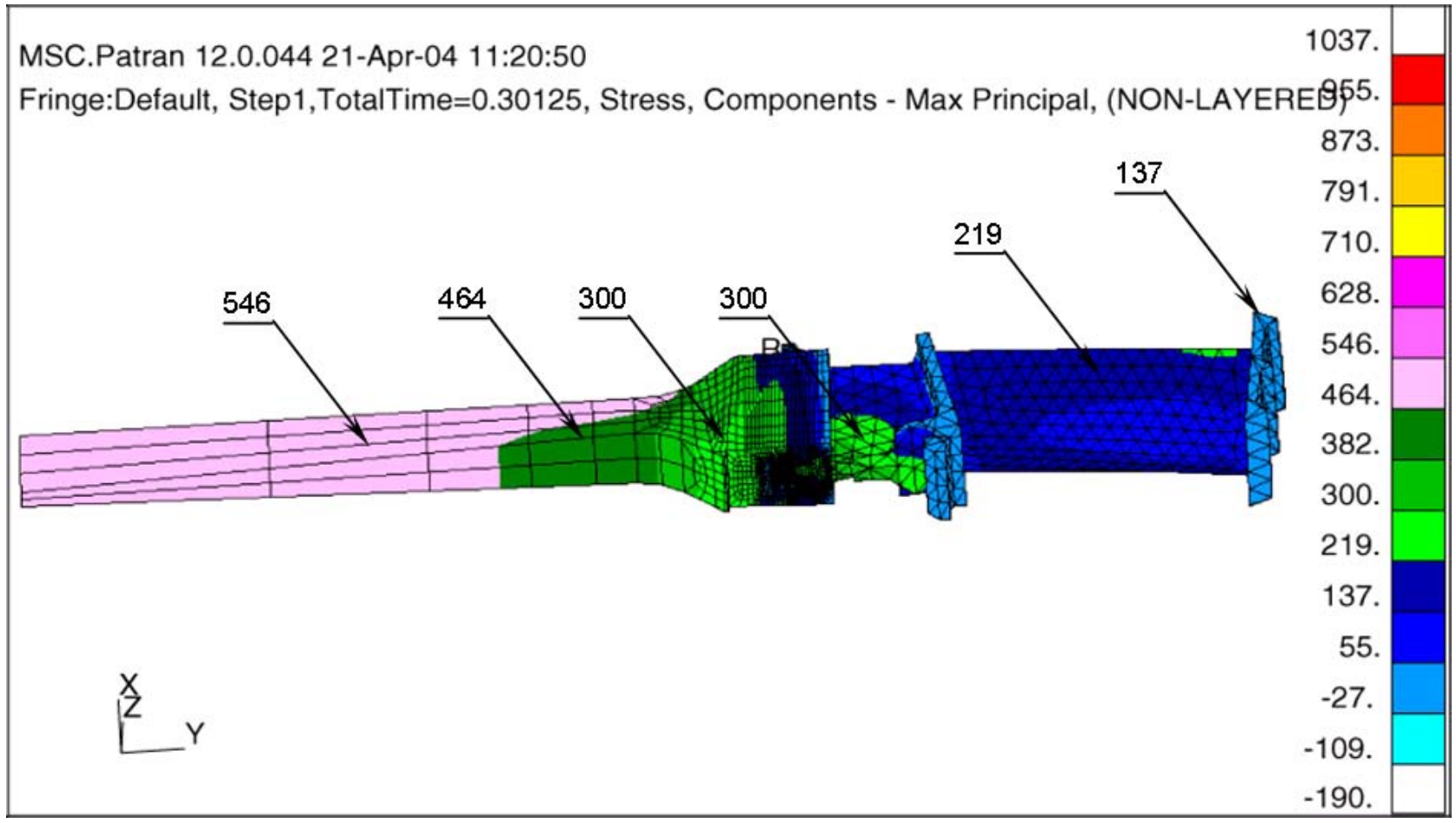

Fig 6. Maximum principal stress distribution of turbine segment under spin rig test 


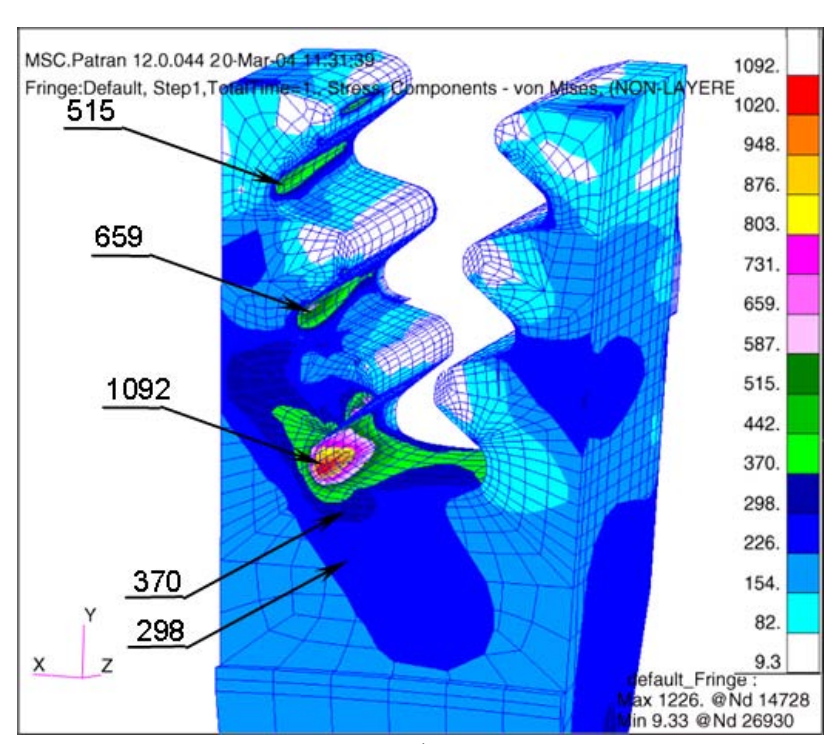

a)

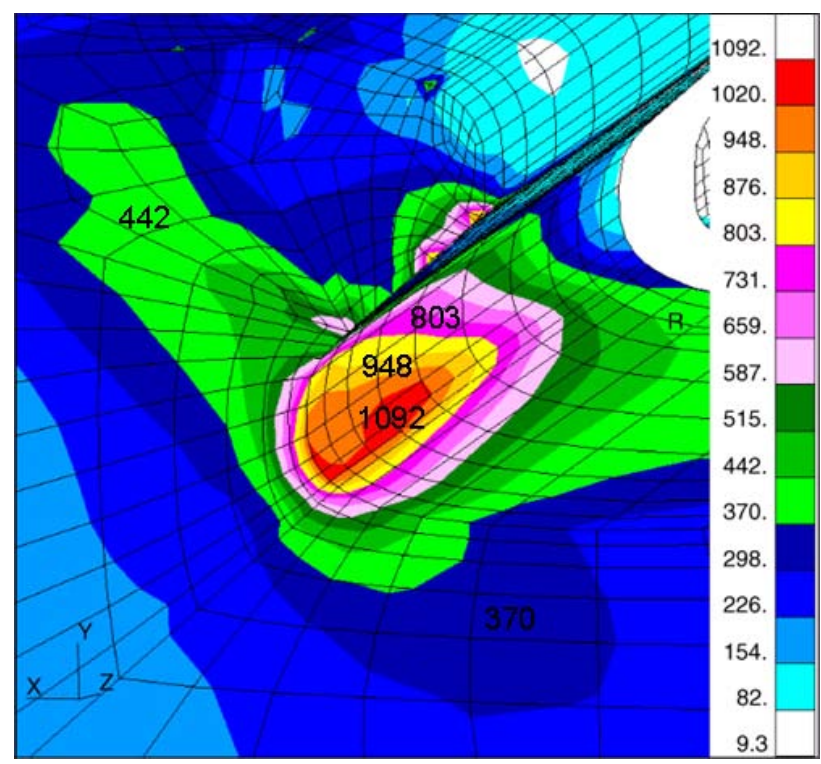

b)

Fig 7. Von Mises stress distribution in the vicinity of the serrated area of disc (a) and magnified view of maximum stress region $(b)$

Figure 6 presents the maximum principal stress distribution of the turbine segment for spin rig test thermo mechanical conditions. As seen from this figure, for the bottom part of the turbine wheel, the stress is about 464 Mpa, while on the blade surfaces the stress is much lower (137-300 MPa).

Figure 7 shows that the area of the maximum Von Mises stress (1092 MPa) for the spin test speed of 14100 rpm is located on the corner of the lowest fir-tree slot of the disc. The high level of stress occurring in this area could be the reason that the first fatigue crack begins in this region of the disc. This zone should be inspected with peculiar attention during the spin test of a turbine.

A considerably lower value of stress $(871 \mathrm{MPa})$ in the area of the fir-tree slots of blade can be observed in Figure $8 \mathrm{a}$. The remaining zones of the blade are not as highly loaded as the serration region.
Figure $8 \mathrm{~b}$ shows the radial displacement distribution for the disc/blade segment. As seen from this figure, the displacement of top part of the blade under a test speed of $14100 \mathrm{rpm}$ is $0,346 \mathrm{~mm}$.

Results clearly show that under performing a spin rig test, attention should be mainly devoted to fir-tree area of the disc and blade, where high stresses occur and the first fatigue crack can be expected.

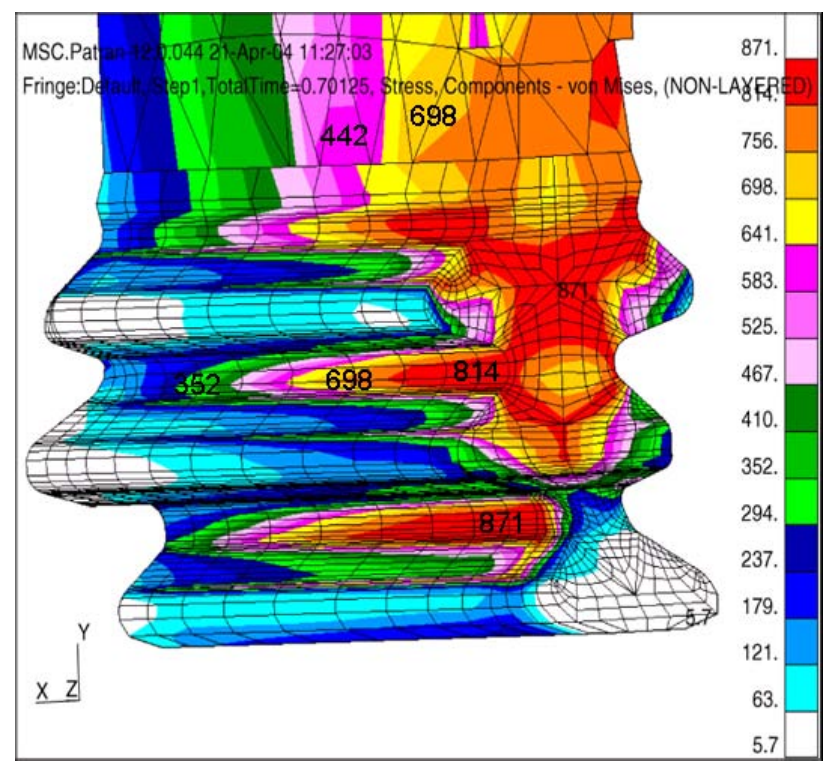

a)

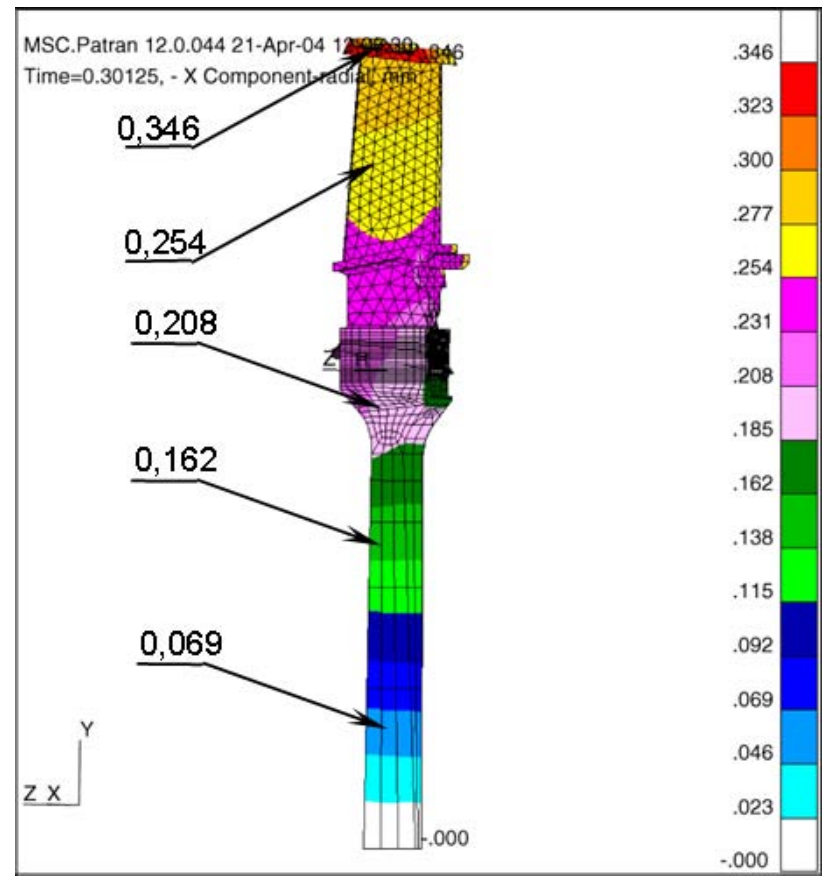

b)

Fig 8. Von Mises stress distribution in the fir-tree slots of blade (a) radial displacement distribution for disc/blade segment (b)

\section{Conclusions}

This paper presents the results of the static calculation of a turbine segment subjected to a spin rig test. To solve the problem, the analysis of the finite element of a complex geometrical model of a disc and 
blade with much nonlinearity such as contact and plasticity was carried out. In this analysis, the stress and displacement contours of a turbine subjected to rotation at constant temperature were obtained. The analysis performed for spin rig conditions showed that the first fatigue cracks can be expected during the spin test in a few critical regions of turbine.

The first critical area of a turbine is located on the corner of third lowest fir-tree slot of the disc, where the maximum stress was observed. Another dangerous zone of a turbine is the serration-fitting region of a blade, where high stress also occurs. The results and their interpretation presented in this study contribute to a better understanding of the mechanical phenomena occurring in jet engine turbines.

\section{References}

1. ABAQUS User's Manual: Ver. 6.4. - Abaqus Inc., 2003.

2. Aerospace Structural Metals Handbook. CRDA/Purde University, 2004. - Ed. 37. - Vol. 5.

3. Bhaumik S.K. Failure of turbine rotor blisk of an aircraft engine // Engineering Failure Analysis. -2002. - Vol 9. - P. 287-301.

4. Chan S.K., Tuba I.S. A finite element method for contact problems of solid bodies / Applications to turbine blade fastenings // International Journal of Mechanical Sciences. - 1971. - Vol 13, Part II. - P. 627-639.

5. Hou J., Wicks B.J. Antoniou R.A. An investigations of fatigue failures of turbine blades in a gas turbine engine by mechanical analysis // Engineering Failure Analysis. - 2002. - Vol 9. - P. 201-211.

6. McEvily A.J. Failures in inspection procedures: case studies // Engineering Failure Analysis. - 2004. - Vol 11. - P. 167-176.

7. Masataka M. Root and groove contact analysis for steam turbine blades // Japan Society of Mechanical Engineering International Journal. - 1992. - Vol. 35, No 4. - P. 508-514.

8. Meguid S.A., Kanth P.S., Czekanski A. Finite element analysis of fir-tree region in turbine discs // Finite Element in Analysis and Design. - 2000. - Vol. 35. - P. 305-317.

9. MSC-PATRAN User's Manual: ver. 2004. - Los Angeles: MSC Corporation, 2004.

10. Park M., Hwang Y., Choi Y., Kim T. Analysis of a J69-T-25 engine turbine blade fracture // Engineering Failure Analysis. - 2002. - Vol 9. - P. 593-601.

11. Papanikos P., Meguid S.A., Stjepanovic Z. Threedimensional nonlinear finite element analysis of dovetail joints in aero-engine discs // Finite Element in Analysis and Design. - 1998. - Vol. 29. - P. 173186.

12. Zboinski G. Physical and geometrical non-linearity's in contact problems of elastic turbine blade attachments // Journal of Mechanical Engineering Sciences. - 1995. - Vol. 209, No 4. - P. 273-286.

13. Zhuang W.Z. Prediction of crack growth from bolt holes in a disc // International Journal of Fatigue. 2000. - Vol. 22. - P. 241-250. 